\title{
High-efficiency time-reversed ultrasonically encoded optical focusing using a large-area photorefractive polymer
}

Yuta Suzuki, Puxiang Lai, Xiao Xu, Lihong V. Wang

Yuta Suzuki, Puxiang Lai, Xiao Xu, Lihong V. Wang, "High-efficiency timereversed ultrasonically encoded optical focusing using a large-area photorefractive polymer," Proc. SPIE 8581, Photons Plus Ultrasound: Imaging and Sensing 2013, 85811G (4 March 2013); doi: 10.1117/12.2005021

SPIE. Event: SPIE BiOS, 2013, San Francisco, California, United States 


\title{
High-efficiency time-reversed ultrasonically encoded optical focusing using a large-area photorefractive polymer
}

\author{
Yuta Suzuki*, Puxiang Lai, Xiao Xu, and Lihong Wang* \\ Optical Imaging Laboratory, Department of Biomedical Engineering, \\ Washington University in St. Louis, St. Louis, Missouri, 63130, USA
}

\begin{abstract}
Time-reversed ultrasonically encoded (TRUE) optical focusing focuses light beyond one transport mean free path by phase-conjugating the ultrasonically tagged light. However, in previous works, only a small portion of the tagged light was phase-conjugated by using a photorefractive $\mathrm{Bi}_{12} \mathrm{SiO}_{20}$ crystal, due to its small active area $\left(1 \times 1 \mathrm{~cm}^{2}\right)$. In this work, we report high-efficiency TRUE focusing using a large-area photorefractive polymer $\left(5 \times 5 \mathrm{~cm}^{2}\right)$, which demonstrated $\sim 40$ times increase in focused energy. Further, we imaged absorbers embedded in a turbid sample of thickness of $\sim 12$ transport mean free paths.
\end{abstract}

Keywords: optical focusing, ultrasound modulation, optical imaging, phase conjugation, time reversal, photorefractive material.

\section{Introduction}

Localization of optical energy at desired locations inside biological tissue is of essential interest in optical applications for biomedicine. However, beyond one transport mean free path $\left(l_{t}^{\prime}\right)$, delivering optical energy with good spatial control becomes more and more difficult due to the multiple scattering in tissue. One way to overcome the limit is iterative wavefront shaping of the incident beam through maximizing a feedback signal from embedded visible targets [1]. However, this approach is not suitable for many biomedical applications, because the limited speed of iterative calculations suffers from microstructural movement of biological tissue. Further, the use of visible targets is not only invasive, but also limits the focusing locations to predetermined positions.

In addressing the need to concentrate optical energy at the desired positions inside biological tissue beyond $1 l_{t}^{\prime}$, $\mathrm{Xu}$ et al. developed a technique named time-reversed ultrasonically encoded (TRUE) optical focusing [2]. The technique is a two-step method. In its recording phase, a focused ultrasound beam spectrally modulates, or tags, the diffuse light inside a scattering medium. Such tagged photons are selectively recorded as a hologram onto an updatable holographic film outside the sample. In the subsequent readout phase, optical focusing into the medium is achieved by phase conjugating (or time reversing, for monochromatic light) the tagged light. As a phase conjugate mirror (PCM), Xu et al. [2] used a photorefractive $\mathrm{Bi}_{12} \mathrm{SiO}_{20}$ (BSO) crystal. Recently, digital versions of the technique were demonstrated, which employed a complementary-metal-oxide-semiconductor camera and a spatial light modulator to achieve digital optical phase conjugation $[3,4]$. The photorefractive PCM-based analog TRUE method has an important advantage over the digital version in its faster operation speed and finer recordable grating resolution. However, inorganic photorefractive crystals can only be fabricated up to a few centimeters in the transverse dimension, which restricts the diffuse light collection from a turbid sample. Further, there are other inferior figure-of-merits of photorefractive crystals, such as their diffraction efficiency, hologram persistency, and slow response time, that make the analog TRUE method infeasible for in vivo applications.

Recent research on photorefractive polymers (PRPs) shows exciting potential to improve the TRUE focusing ability. Unlike their inorganic counterparts, PRPs can be made with large area $\left(>30 \times 30 \mathrm{~cm}^{2},[5]\right)$. Moreover, choosing

\footnotetext{
* Further author information

Yuta Suzuki,ysuzuk@wustl.edu, 1-314-9359587

Lihong V. Wang, lhwang@,wustl.edu, 1-314- 9356152
}

Photons Plus Ultrasound: Imaging and Sensing 2013, edited by Alexander A. Oraevsky, Lihong V. Wang, Proc. of SPIE Vol. 8581, 85811G · (C) 2013 SPIE · CCC code: 1605-7422/13/\$18 · doi: 10.1117/12.2005021 
proper composites allows high diffraction efficiency, long holographic persistency, and/or fast response times $[6,7,8]$. Here, by using a large-area PRP $\left(\sim 5 \times 5 \mathrm{~cm}^{2}\right)$, we show an increase in delivered optical energy in TRUE focusing over previous BSO crystal. Also, in terms of diffraction efficiency, we show that the experimental photorefractive behaviors of the PRPs and a BSO crystal agree with the theoretical expectations in the case of holographic recording using diffuse tagged light. Finally, by using a PRP, we show a preliminary imaging result from a turbid medium, validating the applicability of PRPs for this technique.

\section{Experimental system}

Operation of the optical setup used in this study is schematically illustrated in Figure 1(a). As in the previous photorefractive phase-conjugation experiments, the optical system has three light beams originating from the same laser source: a sample beam (S), a reference beam (R), and a readout beam ( $\left.\mathrm{R}^{*}\right)$. The frequency of $\mathrm{S}$ was adjusted to $2 \mathrm{MHz}$ higher than original laser output by the sequential acousto-optic modulators.

The hologram of the ultrasound-modulated light was recorded, and then its wavefront was reconstructed in the holographic readout phase. In the 800-ms recording phase, S with 3-mm diameter was switched on to illuminate an optically turbid sample which mimics biological tissue. To simulate a biological tissue, we used an intralipid doped gelbased phantom made by dissolving porcine gelatin into water and then adding intralipid to the mixture before it solidified. A focused ultrasound beam with $1-\mathrm{MPa}$ focal pressure was generated by a 2-MHz transducer (Sonic

(a)

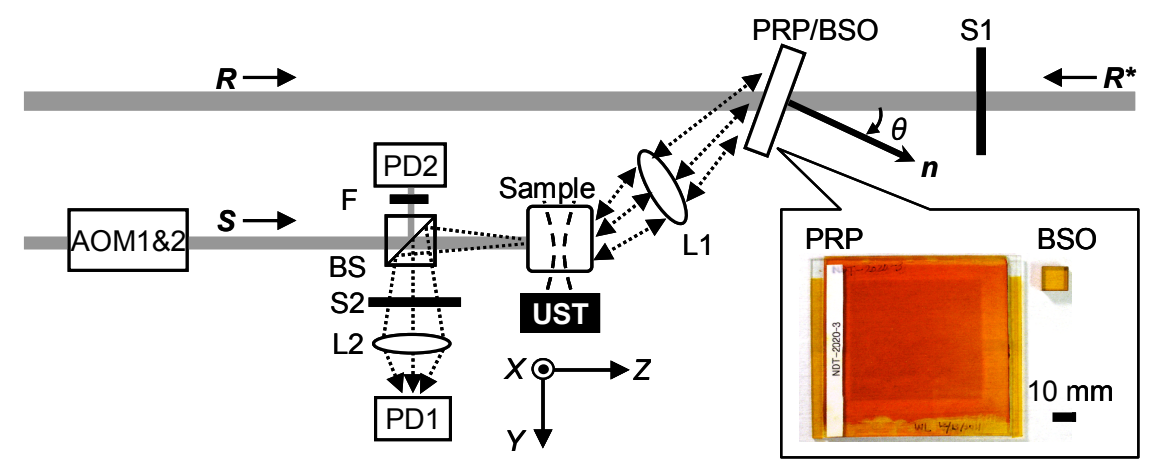

(b)

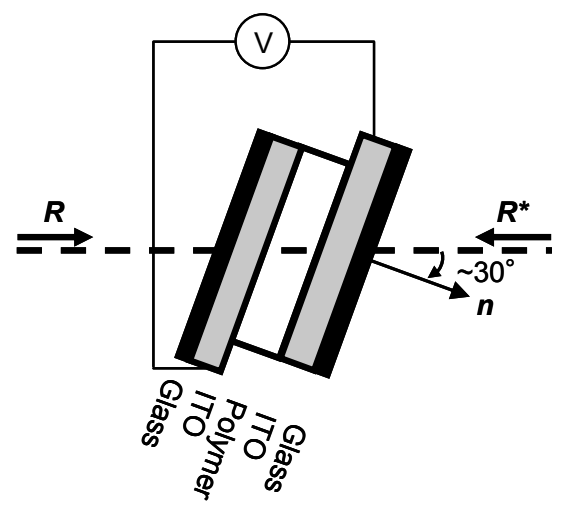

Figure 1. (a) Schematic of the optical setup used in this study. The back surface normal (n) of the PR materials was rotated by angle $\theta$. Either a BSO crystal or PRP was used as the PCM. HWP $i$, ith halfwave plate; PBS $i$, ith polarizing beamsplitter; $\mathrm{M} i$, $i$ th mirror; $\mathrm{AOM} i$, ith acousto-optic modulator; $\mathrm{BS}$, beamsplitter; $\mathrm{S} i, i$ th shutter; $\mathrm{BE} i$, ith beam expander; UST, ultrasound transducer; $L i$, $i$ th lens; F, neutral density filter. $X$ is the sample scanning axis, $Y$ is the acoustical axis, and $Z$ is the optical axis. The inset shows a photograph of the PRP and BSO crystal, demonstrating the advantageously greater area of the PRP. (b) Schematic of high-voltage application to PRP. The voltage was applied across the indium-tinoxide (ITO) coated glass electrodes, which sandwiched the $100-\mu \mathrm{m}$ thick polymer film. The PRP's surface normal was horizontally tilted from the optical axis by $\theta \sim 30$ degrees. 
Concepts, H106) to spectrally modulate the diffuse light propagating inside the sample. The diffuse light exiting the back of the sample (hereafter called "scattered S") was collected to illuminate the photorefractive material. The reference beam $\mathrm{R}$ had a $3-\mathrm{cm}$ diameter. The beam angle between the scattered $\mathrm{S}$ and $\mathrm{R}$ was approximately $20^{\circ}$. $\mathrm{R}$ formed a stationary interference pattern only with the ultrasound-modulated light in the scattered $\mathrm{S}$, which contributed to a hologram, while the unstationary interference patterns between $\mathrm{R}$ and the other frequency components in the scattered $\mathrm{S}$ resulted in a uniform background. Thus, the information of the tagged-light wavefront was selectively recorded in the photorefractive material. Once S was switched off after the holographic recording, shutter S2 was opened to start the readout phase. Then shutter S1 was opened to turn on $50-\mathrm{ms} \mathrm{R} *$ pulse. $\mathrm{R}^{*}$ is a counter propagating reference beam with a 3-cm diameter. Through holographic diffraction, $\mathrm{R}^{*}$ generated the phase-conjugated light $\left(\mathrm{S}^{*}\right)$ of the tagged light from the photorefractive material. After transmitting through the turbid sample for the second time, $\mathrm{S}^{*}$ was partially split by a beamsplitter and detected as the TRUE signal. The repetition rate of the cycle was $1 \mathrm{~Hz}$.

As a photorefractive material that serves as the updatable holographic film, we used either a PRP film, supplied by Nitto Denko, or a BSO crystal as used in our previous experiments. The composite of the PRP film was the same as the polymer device reported in [7]. The transverse dimensions of the PRP film and the BSO crystal were $5 \times 5 \mathrm{~cm}^{2}$ and $1 \times 1 \mathrm{~cm}^{2}$, respectively, as shown in the inset of Figure 1(a). As seen, the PRP film offers an advantageous large area compared to a BSO crystal, which increased collection of the diffuse light from the turbid sample. The PRP film has a thickness of $100 \mu \mathrm{m}$ and is sandwiched between two indium-tin-oxide coated glass slides, while the BSO crystal has a thickness of $0.5 \mathrm{~cm}$. We applied $4-\mathrm{kV}$ dc voltage across the front and back surfaces of the PRP film to enable photorefractivity, as shown in Fig. 1(b). The surface normal of the PRP film was tilted by $\sim 30^{\circ}$ from the propagation direction of $\mathrm{R}$ to increase the diffraction efficiency from the recorded gratings. Although such a tilted configuration of the film reduces its usable transverse area by a factor of $\cos (\theta)$, where $\theta$ is defined in Fig. 1(a), the effective area of the polymer was $\sim 21 \mathrm{~cm}^{2}$, still far exceeding the area of a BSO crystal of $1 \mathrm{~cm}^{2}$. Under our experimental conditions, the measured TRUE signal reached its steady state value after $\sim 5 \mathrm{~s}$, which potentially can be shortened by increasing the intensity of the two writing beams (scatterd S and R). When we used the BSO crystal, $\theta$ was $\sim 10^{\circ}$, and we applied an 8$\mathrm{kV}_{\text {peak-to-peak }}$ square wave electric field at $2.1 \mathrm{kHz}$ across the surface of the crystal as in the previous works [2, 9, 10], to increase the holographic diffraction efficiency [11]. Whenever we replaced PR materials, we adjusted mirrors M4 and M5 to maximize the TRUE signal at the photodetector before taking any measurement.

\section{Theory}

To examine the diffraction efficiencies of PRPs and BSO crystals, it is helpful to compare the experimental results with the expected behaviors in theory. According to the theory of photorefractive crystals, the diffraction efficiency of a recorded grating is proportional to the contrast (or modulation) squared of the interference grating, when the two writing beams are plane waves and the reference beam is much stronger than the signal beam. In the case of TRUE focusing, however, the problem is less trivial, because the signal beam is the diffuse tag light which is buried in a strong untagged background light.

To extend the theory to the case of diffuse and tagged signal beams, let us denote the complex amplitude of the plane reference beam $\mathrm{R}$ as $\mathrm{E}_{\mathrm{R}}\left(f_{0}\right)$, that of the unmodulated scattered $\mathrm{S}$ as $\mathrm{E}_{\mathrm{S}}\left(f_{0}-f_{\mathrm{a}} ; x, y\right)$, and that of the tagged light as $\mathrm{E}_{\mathrm{T}}\left(f_{0} ; x, y\right)$. Note that the spatial dependency of the scattered light, and the frequency associated with each of the electrical fields are explicitly spelled out inside the brackets, where $f_{0}$ is the original laser output frequency, and $f_{\mathrm{a}}$ is the acoustic frequency applied to the sample. Then, the complex amplitude of the electrical field that impinges on the photorefractive material may be written as

$$
E(x, y)=E_{\mathrm{R}}\left(f_{0}\right)+E_{S}\left(f_{0}-f_{a} ; x, y\right)+E_{T}\left(f_{0} ; x, y\right) .
$$

Therefore, the time-averaged intensity distribution can be expressed as the square of eq. (1), and becomes

$$
I(x, y)=\left|E_{R}\right|^{2}\left\{1+\frac{\left|E_{s}\left(f_{0}-f_{a} ; x, y\right)\right|^{2}}{\left|E_{R}\right|^{2}}+m \frac{\operatorname{Re}\left[E_{R}^{*}\left(f_{0}\right) E_{T}\left(f_{0} ; x, y\right)\right]}{\left|E_{R}\right|\left|E_{T}\left(f_{0} ; x, y\right)\right|}\right\},
$$

where cross talks between the electrical fields of different frequencies are dropped, by taking the time average of the intensity. Also, by assuming that the tagged light is much weaker than $\mathrm{R}$, its contribution to the offset of the interferogram is neglected. $m$ in eq. (2) is the local contrast (or modulation) of the hologram, defined by

$$
m(x, y)=\left|\frac{E_{T}\left(f_{0} ; x, y\right)}{E_{R}}\right| .
$$


For simplicity, let us assume that $\left|E_{R}\right|^{2}>\left|E_{S}\right|^{2}$, and neglect the second term in (2). This condition can be easily met in the real experiment, since only a small portion is collected as the scattered $\mathrm{S}$ after the diffuse medium. Following the knowledge of photorefractive diffraction efficiency, we expect that the diffraction efficiency should be proportional to the $m(x, y)$ squared, $\left|E_{\mathrm{T}}\left(f_{0} ; x, y\right)\right|^{2} /\left|\mathrm{E}_{\mathrm{R}}\right|^{2}$, which is the local intensity ratio of tagged light to $\mathrm{R}$. In the experiment, we measure the spatially averaged diffraction efficiency of the hologram, which is our interest. By taking the spatial average of $m(x, y)$, we see that the holographic diffraction efficiency is proportional to the average intensity ratio of tagged light to R. Further, because $E_{\mathrm{T}}$ is proportional to the scattered $\mathrm{S}$, the diffraction efficiency of the hologram is expected to be proportional to the intensity ratio of the scattered $\mathrm{S}$ to $\mathrm{R}$, when $\left|E_{\mathrm{R}}\right|^{2}>\left|E_{\mathrm{S}}\right|^{2}$.

\section{Experimental Results}

To see the phase-conjugation performance by using a PRP film as a PCM instead of a BSO crystal, we compared the R-to-signal conversion efficiency for both of the scenarios. In the first experiment, we fixed the S intensity while increasing both the $\mathrm{R}^{*}$ and $\mathrm{R}$ intensities at the fixed $\mathrm{R}^{*}$-to- $\mathrm{R}$ intensity ratio of 17 , and observed the changes in the TRUE signal intensities. The used sample was a 1-cm-thick gelatin-intralipid sample having a reduced scattering coefficient $\mu_{\mathrm{s}}^{\prime}=5 \mathrm{~cm}^{-1}$. The diffraction efficiency was calculated by dividing the peak intensity of the TRUE signal by that of $R^{*}$. Figure 2(a) shows the measured variations of the diffraction efficiencies for the different intensity ratios of

(a)

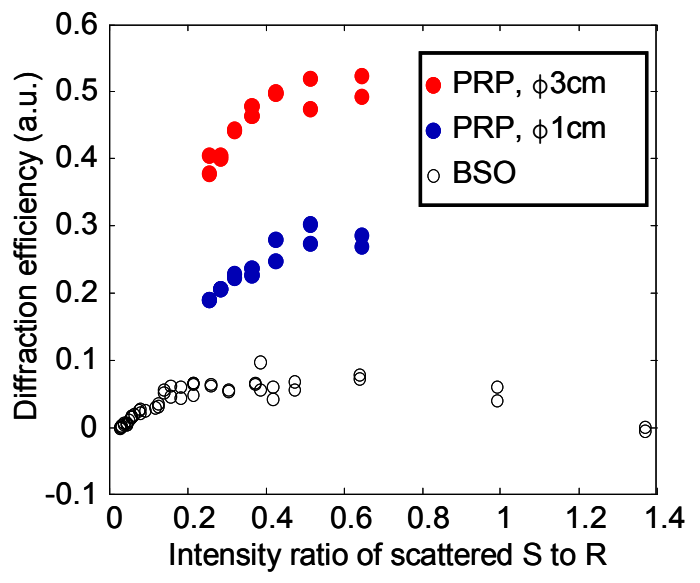

(c)

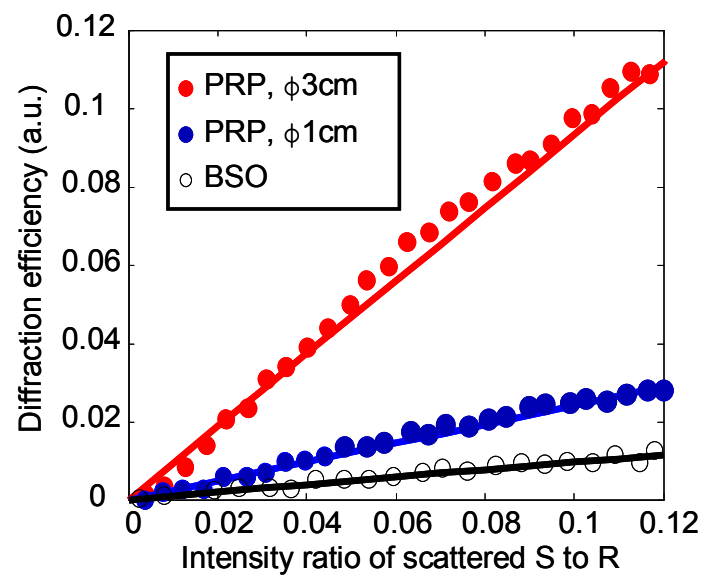

(b)

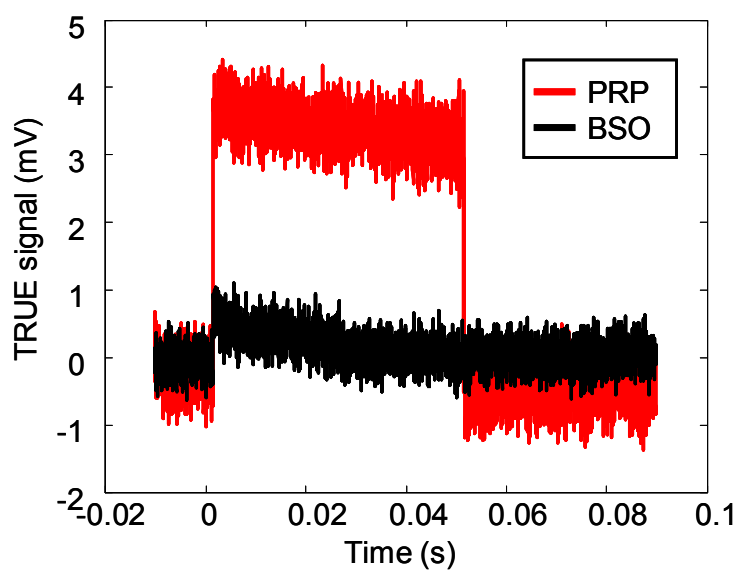

(d)

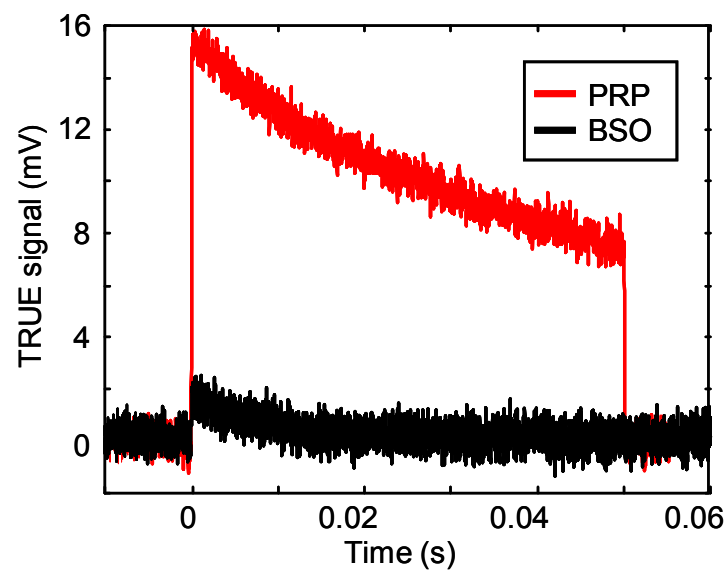

Figure 2. (a) Plot of diffraction efficiencies versus intensity ratio of scattered S to R for PRP1. (b) Representative temporal profiles of TRUE signals acquired using the PRP1 and BSO crystal. (c) Plot of diffraction efficiencies versus intensity ratio of scattered S to R for PRP2. Linear fitting curves are also shown. (d) Representative temporal profiles of TRUE signals acquired using the PRP2 and BSO crystal. 
the scattered $\mathrm{S}$ to $\mathrm{R}$ in both scenarios, by using a PRP (PRP1). We see that, when we limited the polymer aperture size to $1-\mathrm{cm}$ diameter, which is comparable to the area of the BSO crystal, the diffraction efficiency increased by $\sim 3$ times over that of the BSO crystal. Moreover, when we increased the aperture size to $3-\mathrm{cm}$ diameter, the efficiency increased by $\sim 5$ times. The improvement by increasing the aperture size is not the same as the increment of the PRP area, because of the non-uniform photorefractive properties of PRP over its area. It was expected to see a linear relationship when the intensity ratio of scattered $\mathrm{S}$ to $\mathrm{R}$ is small enough, but while conducting the experiment, the dielectric breakdown of the PRP1 prohibited the measurement. The strong $\mathrm{R}$ and $\mathrm{R}^{*}$ intensities led to the dielectric breakdown, which was used to create a low intensity ratio of scattered $\mathrm{S}$ to R. Figure 2(b) shows TRUE signal waveforms acquired by using a BSO crystal and the PRP1 under the same conditions of R and $\mathrm{R}^{*}$. As seen in Fig. 2(b), PRP offered a stronger signal peak for a longer duration.

By using another PRP (PRP2), we conducted a further experiment to see the linear relationship when R is much stronger than scattered $\mathrm{S}$ intensity. To prevent the polymer damage caused by the strong $\mathrm{R}$ and $\mathrm{R}^{*}$ intensities, we varied the $\mathrm{S}$ intensity while fixing $\mathrm{R}$ and $\mathrm{R}^{*}$ intensities at $10 \mathrm{~mW} / \mathrm{cm}^{2}$ and $140 \mathrm{~mW} / \mathrm{cm}^{2}$, respectively. In this way, low intensity ratio of scattered $\mathrm{S}$ to $\mathrm{R}$ can be achieved when the $\mathrm{S}$ intensity is low enough, without causing dielectric breakdown of the polymer. The result is shown in Figure 2(c), where we see the explained linear relationship of the photorefractive diffraction efficiencies. Using PRP2, we increased the diffraction efficiency by $\sim 2$ times with an aperture size of 1-cm diameter, and $\sim 8$ times with an aperture size of $3-\mathrm{cm}$ diameter. A pair of signal waveforms obtained by using the BSO crystal and PRP2 is shown in Figure 2(d), which further validates the advantages of PRP of longer persistency and $\sim 8$ times higher diffraction efficiency.

Further, to demonstrate the PRP applicability for TRUE focusing, we obtained images from a turbid phantom, by embedding two absorbers at its middle plane. The sample had the reduced scattering coefficient $\mu_{\mathrm{s}}{ }^{\prime}=12 \mathrm{~cm}^{-1}$, and the geometrical thickness $d=1 \mathrm{~cm}$, which is equivalent to $\sim 1.2 \mathrm{~cm}$ human tissue. The photograph of the sample middle
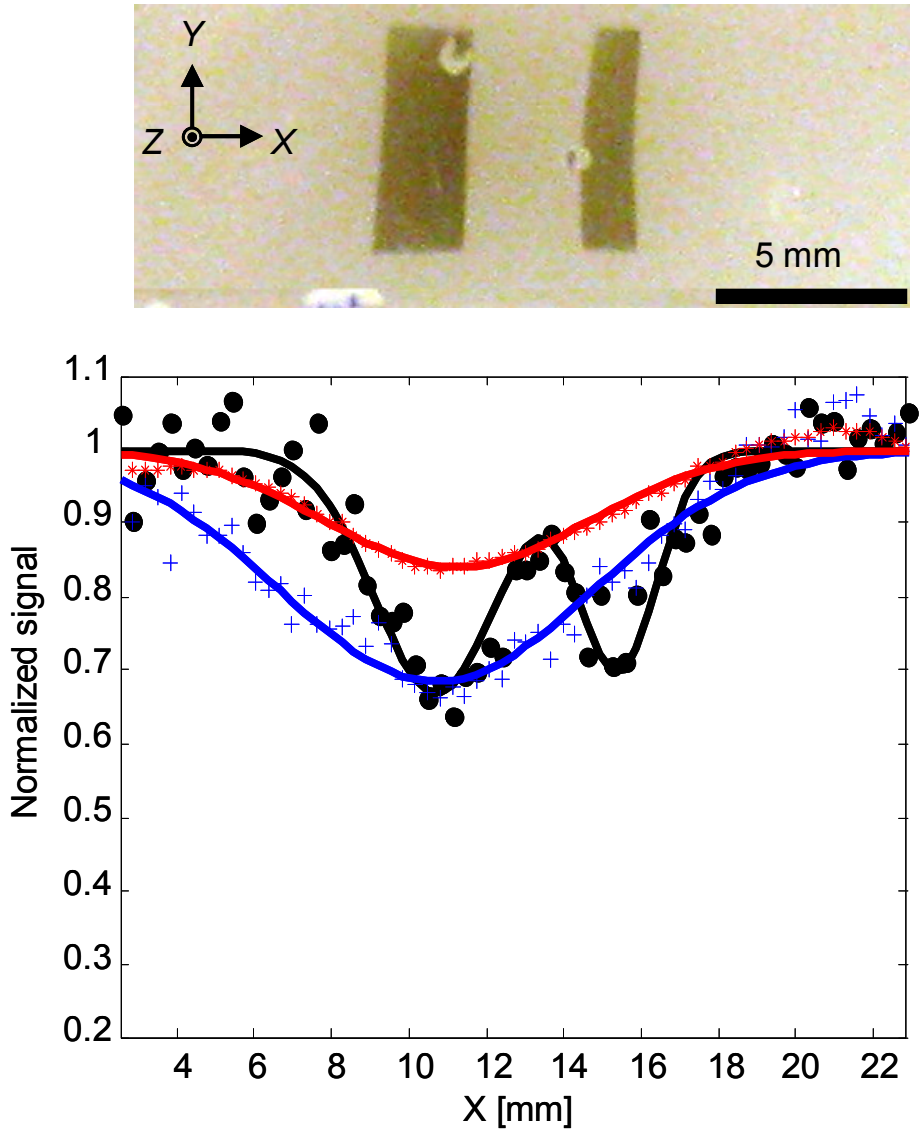

Figure 3. 1-D image demonstrating TRUE optical focusing using PRP2. (a) Photograph of the imaging plane, which was set middle plane of the sample. (b) Normalized TRUE signal, TRDC signal, and DC signal versus the $X$ axis. 
plane that contained the two absorbers is shown in Figure 3(a). We scanned the sample in the X direction and the TRUE signal was averaged for 32 times at each sample position. We also acquired the image by using the scattered S intensity from the sample when the ultrasound is off (DC image), and by using the time-reversed dc light (TRDC image). The sample scanning step size was $0.32 \mathrm{~mm}$. As seen in Figure 3(b), the TRUE image resolved the two absorbers embedded inside the sample, while the DC and TRDC images did not resolve the absorbers, which validates the TRUE focusing ability by using PRP2.

\section{Discussion and Conclusions}

Towards in vivo TRUE focusing, there are several issues that must be addressed. First, the slow photorefractive response time of $\sim 5 \mathrm{~s}$ is an issue because of the fast speckle decorrelation time of tissue $(\sim \mathrm{ms})$. Such fast photorefractive performance is possible by using PRPs with different composites, and by short pulse lasers, as reported in some recent works on PRPs [8]. Second, we noticed that phase-conjugation performance of our PRP fluctuated over a long time period ( $\sim 30 \mathrm{~min})$. This issue also may be addressed by using fast polymers, by shortening the measurement of imaging experiments. Also, using the two-wave mixing signal as a reference signal to normalize the acquired phase-conjugated signal is another approach. Thirdly, our PRP had a relatively lower damage threshold compared to that of a BSO crystal. By expanding the $\mathrm{R}$ and $\mathrm{R}^{*}$ beam, we can overcome this issue while maintaining the same optical powers for both beams and observable signal strength.

In summary, we showed a phase-conjugation efficiency increase in TRUE focusing by using a PRP. Also, for the diffuse tagged light case, we showed that diffraction efficiencies of PRPs and a BSO crystal match the presented photorefractive theory. Further, using a PRP, we resolved two optical absorbers embedded in a turbid medium with $\mu_{\mathrm{s}}{ }^{\prime}=$ $12 \mathrm{~cm}^{-1}$, and geometrical thickness $d=1 \mathrm{~cm}$, which is equivalent to $1.2-\mathrm{cm}$-thick human tissue. Although there are several difficulties for in vivo applications, it is likely to be able to solve these step-by-step. The presented efficiency increase of TRUE focusing is an encouraging step towards the future applications of this technique.

\section{References}

[1] Vellekoop, I. M., van Putten, E. G., Lagendijk, A., and Mosk, A. P, "Demixing light paths inside disordered metamaterials," Opt. Express 16, 67-80 (2008)

[2] Xu, X., Liu, H., and Wang, L. V., "Time-reversed ultrasonically encoded optical focusing into scattering media," Nature Photon. 5, 154-157 (2011)

[3] Si, K., Fiolka, R., and Cui, M., "Fluorescence imaging beyond the ballistic regime by ultrasound-pulse-guided digital phase conjugation," Nature Photon, 6, 657-661 (2012)

[4] Wang, Y. M., Judkewitz, B., DiMarzio, C. A., and Yang, C., "Deep-tissue fluorescence imaging with digitally time-reversed ultrasound-encoded light," Nature Commun, 3, 928 (2012)

[5] Blanche, P. -A., Bablumian, A., Voorakaranam, R., Christenson, C., Lin, W., Gu, T., Flores, D., Wang, P., Hsieh, W. -Y., Kathaperumal, M., Rachwal, B., Siddiqui, O., Thomas, J., Norwood, R. A., Yamamoto, M., and Peyghambarian, N., "Holographic three-dimensional telepresence using large-area photorefractive polymer," Nature, 468(4), 80-83 (2010)

[6] Meerholz, K., Volodin, Sandalphon, B. L.,, Kippelen, B., and Peyghambarian, N., "Photorefractive polymer with high optical gain and diffraction efficiency near 100\%," Nature, 371, 497-500 (1994)

[7] Tay, S., Blanche, P. A., et al., "An updatable holographic three-dimensional display," Nature, 451(7179), 694$698(2008)$

[8] Eralp, M., Thomas, J., Tay, S., Li, G., Schülzgen, A., "Submillisecond response of a photorefractive polymer under single nanosecond pulse exposure," Appl. Phys. Lett., 89, 114105 (2006)

[9] Lai, P., Xu, X., Liu, H., Suzuki, Y., and Wang, L. V., "Reflection-mode time-reversed ultrasonically encoded optical focusing into turbid media," Journal of Biomedical Optics, 16(8), 080505 (2011)

[10] Liu, H., Xu, X., Lai, P., and Wang, L. V., "Time-reversed ultrasonically encoded optical focusing into tissuemimicking media with thickness up to 70 mean free paths," Journal of Biomedical Optics, 16(8), 086009 (2011)

[11] Stepanov, S. I., and Petrov, M. P., "Efficient unstationary holographic recording in photorefractive crystals under and external alternating electric field," Optics Communications, 53(5), 292-295 (1985) 